Tropical Journal of Pharmaceutical Research July 2016; 15 (7): 1353-1360

ISSN: $1596-5996$ (print); 1596-9827 (electronic)

(C) Pharmacotherapy Group, Faculty of Pharmacy, University of Benin, Benin City, 300001 Nigeria.

All rights reserved.

Available online at http://www.tjpr.org

Original Research Article

http://dx.doi.org/10.4314/tjpr.v15i7.1

\title{
Development of docetaxel and alendronate-loaded chitosan-conjugated polylactide-co-glycolide nanoparticles: In vitro characterization in osteosarcoma cells
}

\author{
Ya-Feng Liu*, Rui Liu, Xue-Yang Li, Zhen Song and Xue-Hang Zhao \\ Department of Orthopedics, KaiFeng Central Hospital, Henan 475000, PR China \\ *For correspondence: Email: liuyafeng68@hotmail.com; Tel/Fax: +8637125550069
}

Received: 10 February 2016

Revised accepted: 18 June 2016

\begin{abstract}
Purpose: To develop docetaxel (DTX)- and alendronate (ALN)-loaded, chitosan (CS)-conjugated polylactide-co-glycolide (PLGA) nanoparticles (NPS) to increase therapeutic efficacy in osteosarcoma cells. Methods: Drug-loaded PLGA NPs were prepared by nanoprecipitation and chemically conjugated by the carboxylic group of PLGA to the amine-bearing CS polymer. The nanocarrier was characterized by dynamic light scattering, transmission electron microscopy, scanning electron microscopy, and differential scanning calorimetry as well as by in vitro drug release and cell culture studies.

Results: NP size was within the tumour targeting range ( $200 \mathrm{~nm})$ with an effective positive charge (20 $m V)$, thus increasing cellular uptake efficiency. Morphological analysis revealed clear spherical particles with uniform dispersion. The NPs exhibited identical sustained release kinetics for both DTX and ALN. CS-conjugated PLGA with dual-drug-loaded (DTX and AL) NPs showed typical time-dependent cellular uptake and also displayed superior cytotoxicity in MG-63 cells compared with blank NPs, which were safe and biocompatible.

Conclusion: Combined loading of DTX and ALN in NPs increased the therapeutic efficacy of the formulation for osteosarcoma treatment, thus indicating the potential benefit of a combinatorial drug regimen using nanocarriers for effective treatment of osteosarcoma.
\end{abstract}

Keywords: Chitosan, Docetaxel, Alendronate, Nanocarriers, Sustained-release kinetics, Polylactide-coglycolide, Osteosarcoma, Cellular uptake

Tropical Journal of Pharmaceutical Research is indexed by Science Citation Index (SciSearch), Scopus, International Pharmaceutical Abstract, Chemical Abstracts, Embase, Index Copernicus, EBSCO, African Index Medicus, JournalSeek, Journal Citation Reports/Science Edition, Directory of Open Access Journals (DOAJ), African Journal Online, Bioline International, Open-J-Gate and Pharmacy Abstracts

\section{INTRODUCTION}

Osteosarcoma is the most prevalent malignant bone cancer. It commonly occurs in children and adolescents: approximately 400 cases per year are diagnosed in the United States alone [1]. Osteosarcoma mostly arises in the metaphyseal ends of long bones, and is characterized by malignant metastatic tumours that differentiate as cartilage and bone [2]. The survival rate is nearly $20 \%$ with surgery; however, it increases to $70 \%$ when combined with anticancer drug therapy. Thus, surgery and chemotherapy play a vital role in the treatment of osteosarcoma. A recently multi-drug regimen reportedly improved osteosarcoma treatment [3].

Combination therapy has been considered a promising strategy for improving therapeutic efficiency and minimising side effects [4]. The combination of two or more chemotherapeutic drugs may act synergistically in cancer cell 
suppression [5,6]. In this study, docetaxel (DTX) and alendronate (ALN) were selected as a unique drug combination for the synergistic treatment of bone cancers.

DTX is a frontline chemotherapeutic agent that is active against a wide spectrum of cancers. DTX acts on the microtubules of the malignant cell to suppress its proliferation, killing cancer cells by inducing a G2/M phase arrest of the cell cycle. Despite excellent therapeutic potential, DTX treatment results in severe adverse effects, including neutropenia, anaemia, and hypersensitivity reactions [7]. ALN is used because of its strong osteoprotective properties and its affinity for bone cancer cells. It belongs to the class of amino bisphosphonates (NBP), and is in the fourth class of the Biopharmaceutics Classification System (BCS) because of its poor solubility and low bioavailability $[8,9]$.

Nanotechnology-based formulation is a promising alternative in cancer treatment. Polylactide-co-glycolide (PLGA) exhibits excellent biocompatibility and biodegradability, and has been extensively used as a carrier for drug delivery [10]. This system extravasates solid tumours into the tumour site by passive targeting via an enhanced permeability and retention effect (EPR). However, the PLGA nanocarrier itself is not sufficient. Surface modification of PLGA with certain hydrophilic polymers enables these nanoparticles (NPs) to increase their selectivity for cellular binding and internalization [11]. For example, chitosan (CS), a biocompatible and nontoxic cationic polysaccharide, is commonly used for surface modifications.

The aim of this study was to develop a CScoated PLGA nanocarrier system for the combined delivery of DTX and ALN to osteosarcoma cells.

\section{EXPERIMENTAL}

\section{Materials}

PLGA-COOH with a lactide to glycolide ratio of 50:50 and an average molecular weight of 15 $\mathrm{kDa}$ was purchased from Ji'nan Daigang Biological Co. Ltd (Shandong, China). CS (deacetylation degree, $>84 \%$ ) was purchased from Zhanjiang Xinmao Chemical \& Glass Company (Zhanjiang, China). DTX, ALN, and 3[4,5-dimethylthiazol-2-yl]-2,5-diphenyltetrazolium bromide (MTT) were purchased from Sigma Chemical Co., USA). All chemicals were analytical grade and used without further purification.

\section{Preparation of CS-coated PLGA NPS containing DTX and ALN}

DTX-loaded PLGA NPs were prepared by solvent diffusion (nanoprecipitation) as previously reported [12]. The organic phase (acetone) containing DTX (15 mg) and ALN (15 mg) and PLGA (200 mg) in a 1:1:1 ratio (v:v) was slowly injected $(0.5 \mathrm{~mL} / \mathrm{min})$ into $20 \mathrm{~mL}$ of aqueous phase containing Poloxamer $188(0.5 \% \mathrm{w} / \mathrm{v})$ as a stabilizer. The resulting NPs were recovered by centrifugation at $25,000 \mathrm{rpm}$ for $30 \mathrm{~min}$ and purified by dialysis against water and lyophilized for $48 \mathrm{~h}$.

The freeze-dried PLGA nanoparticles were dispersed in phosphate buffered saline (PBS) solution ( $\mathrm{pH} \mathrm{6.0)}$ under bath sonication at $37^{\circ} \mathrm{C}$. $\mathrm{N}$-hydroxysuccinimide (NHS) and $\mathrm{N}$-(3dimethylaminopropyl)- $\mathrm{N}^{\prime}$ ethyl carbodiimide hydrochloride $(E D C \cdot \mathrm{HCl})$ was added to activate the carboxyl group of PLGA. Different concentrations of CS were added into the above solution and the reaction was allowed to continue for $24 \mathrm{~h}$. The excess EDC, NHS, and unreacted CS were eliminated by centrifugation. A schematic illustration of the preparation of dualdrug-loaded CS-coated PLGA NPs is shown in Figure 1.

\section{Particle size and zeta potential measurement}

The hydrodynamic diameter of the NPs was measured using a dynamic light scattering (DLS) technique. A Zetasizer Nano ZS (Malvern Instruments, Worcestershire, UK) was used to measure the size at room temperature with an angle of detection of $176^{\circ}$. Zeta potential measurements were performed with a detection angle of $17^{\circ}$ and calculated using the Smoluchowski model. The samples were suitably diluted $(200 \mathrm{mg} / \mathrm{mL})$ with double-distilled water such that the mean count rate was approximately $300 \mathrm{kcps}$.

\section{Morphological characterization}

Morphological studies of the NPs were conducted using transmission electron microscopy (TEM) and field emission scanning electron microscopy (FE-SEM). TEM (H-7600; Hitachi, Japan) was performed by staining a small drop of the NP suspension with a $2 \%$ phosphotungstic acid (PTA) solution. The stained sample was deposited onto a carbon coated copper grid and dried for $30 \mathrm{~min}$ at room temperature. 

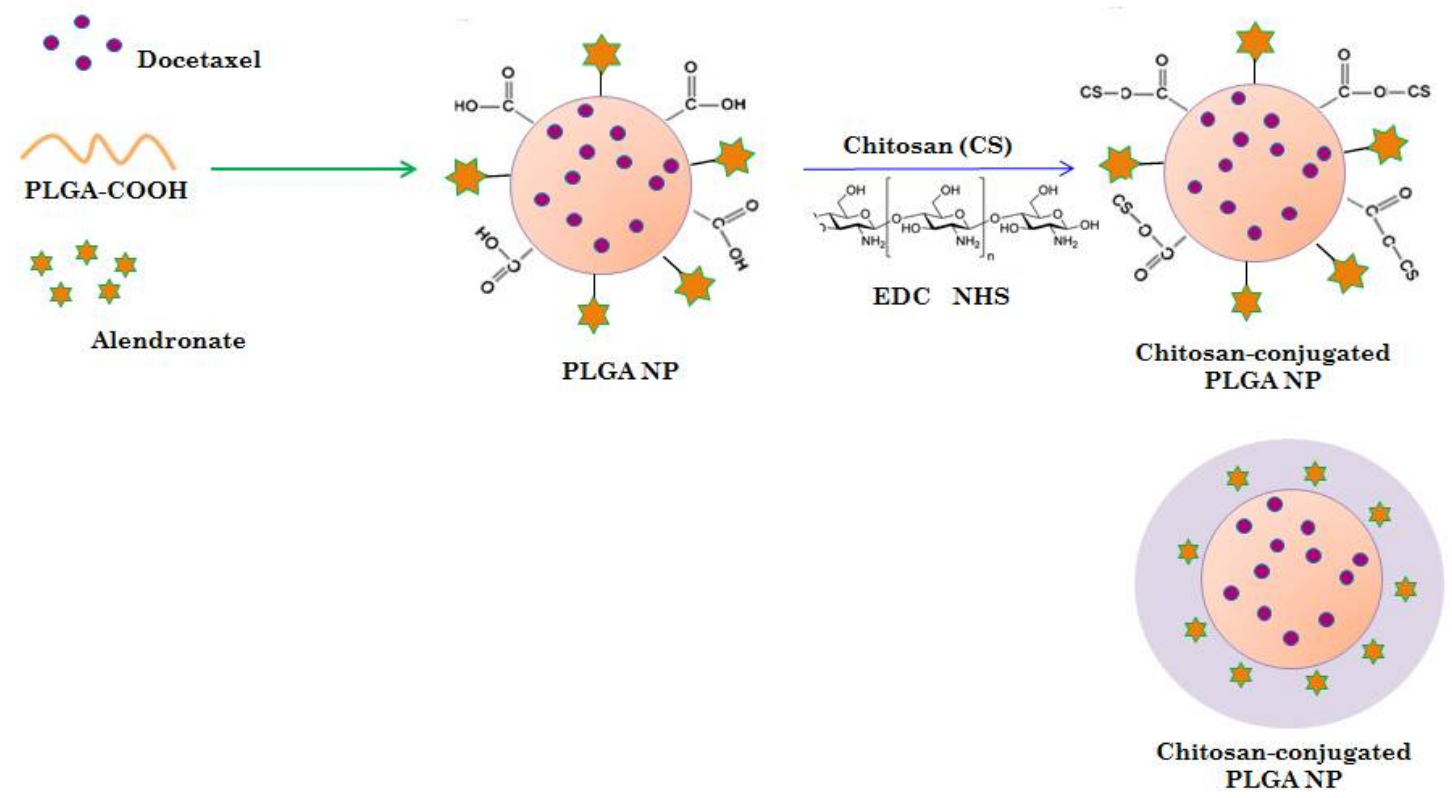

Figure 1: Schematic illustration of the preparation of docetaxel (DTX)- and alendronate (ALN)-loaded chitosan (CS)-conjugated polylactide-co-glycolide (PLGA) nanoparticles

The samples were examined under an acceleration voltage of $100 \mathrm{kV}$ in conventional TEM-mode. FE-SEM was performed with the help of an electron microscope (JSM-7610F scanning electron microscope, Tokyo, Japan). Lyophilized NP samples were reconstituted with deionized water and spread onto a carbon tape over a stub. The samples were vacuum-dried and gold coating was applied using an ion sputtering device. The gold-coated samples were vacuum-dried and examined under an electron microscope.

\section{Differential scanning calorimetry (DSC)}

DSC measurement of ALN, DTX, and PLGAALN-DTX CS was performed using a calorimeter (Mettler TA4000, Mettler Toledo, OH, USA). Empty aluminum pans were used as a reference and samples were carefully placed in another aluminum pan. The measurement was done in an inert atmosphere within a temperature range of $30-200{ }^{\circ} \mathrm{C}$ at $10{ }^{\circ} \mathrm{C} / \mathrm{min}$.

\section{Determination of loading efficiency}

Loading efficiency was calculated from the total amount of drug added versus the amount of drug entrapped in the NPs. Briefly, the drug-loaded complex was pushed through an Amicon centrifugal filter by centrifuging at $5,000 \mathrm{rpm}$ for $10 \mathrm{~min}$. The filtrate was analysed for unentrapped drug using high-performance liquid chromatography (HPLC) at $320 \mathrm{~nm}$ and $230 \mathrm{~nm}$.
Standard curves were individually plotted for ALN and DTX. Loading efficiency (L) was calculated as in Equation 1.

$L(\%)=\{($ Dt Df $) / W\} 100$

where Dt, Df, and W are the total amount of DTX, the amount of free DTX, and the total weight of NPs, respectively.

\section{In vitro release studies}

In order to investigate the release of DTX and ALN from CS-PLGA NPs, a fixed amount (1 $\mathrm{mg} / \mathrm{mL}$ ) of the drug-loaded NPs was incubated in $10 \mathrm{~mL}$ of phosphate buffer solution PBS $\mathrm{pH} 7.4$, ionic strength $0.1 \mathrm{M}$ ) in a dialysis bag (molecular cut-off of $10 \mathrm{kDa}$ ), at $37^{\circ} \mathrm{C}$ with gentle magnetic stirring $(100 \mathrm{rpm})$. At fixed time intervals, $1 \mathrm{~mL}$ of the supernatant was withdrawn and replaced with fresh buffer. The amount of DTX and ALN released in the collected samples was determined by HPLC as described above. All experiments were performed in triplicate.

\section{Cell culture}

MG-63 cells were obtained from American Type Culture Collection (ATCC; Rockville, MD, USA). The cells were grown in Dulbecco's Modified Eagle Media (DMEM) DMEM supplemented with $10 \%$ fetal bovine serum (FBS), 100 units $/ \mathrm{mL}$ penicillin, and $100 \mathrm{mg} / \mathrm{mL}$ of streptomycin. Cells 
were maintained at $37{ }^{\circ} \mathrm{C}$ with $5 \% \mathrm{CO}_{2}$ in a humidified incubator.

\section{Cytotoxicity assay}

Cell viability was used to determine the cytotoxic potential of individual formulations as determined by 3-[4,5-dimethylthiazol-2-yl]-2,5diphenyltetrazolium bromide (MTT) assay. Briefly, cells were seeded into a 96-well plate at a seeding density of $0.5 \times 10^{4}$ cells in $0.1 \mathrm{~mL}$ growth medium and incubated for $48 \mathrm{~h}$. The following day, the cells were treated with the respective formulations (DTX, ALN, DTX-ALN, and DTX-ALN-CS-PLGA NPs) at increasing concentrations from $0.01 \mathrm{mg} / \mathrm{mL}$ to $10 \mathrm{mg} / \mathrm{mL}$. The respective formulations were incubated for $24 \mathrm{~h}$. At each time interval, cells were washed with PBS and treated with MTT solution (5 $\mathrm{mg} / \mathrm{mL}$ in serum-free media) and incubated for another $4 \mathrm{~h}$. The resulting formazan crystals were dissolved in $0.1 \mathrm{~mL}$ of dimethyl sulfoxide (DMSO). The mixture was gently shaken in a microplate reader before measuring the absorbance at $570 \mathrm{~nm}$. Cell viability was calculated after subtracting each value from the control. Each test was carried out 8 times $(n=8)$.

\section{Flow cytometry studies}

MG-63 cells $\left(5 \times 10^{5}\right)$ were seeded in a 6 -well plate and incubated for $24 \mathrm{~h}$ at $37^{\circ} \mathrm{C}$. The cells were treated with PLGA and CS-PLGA NPs for 1 h. The cells were washed 3 times with PBS, trypsinized, centrifuged, resuspended in PBS, and analysed on a fluorescence-activated cell sorting (FACS) flow cytometer (Becton Dickinson, USA).

\section{Statistical analysis}

Student's t-tests were used to determine the differences among groups. The results are presented as mean \pm standard deviation (SD; $n=$ 3) with $p<0.05$ considered statistically significant. Statistical significance was determined using SPSS software (version 16; IBM, United States.

\section{RESULTS}

\section{Characteristics of DTX- and ALN-loaded CS- conjugated PLGA NPs}

In the present study, a unique combination of DTX and ALN was used to treat osteosarcoma cells. For this purpose, CS was conjugated to the carboxylic group of PLGA and loaded with DTX and ALN. The dual-drug-loaded NPs were successfully prepared with high drug entrapment of $83.7 \pm 1.23$ and $71.89 \%$ corresponding to 6.51 and $5.21 \%$ drug loading of DTX and ALN. The particle size of the PLGA NPs (PNP) and CS-coated PLGA NPs (CPNP) was observed to be $165 \pm 1.72$ and $202 \pm 4.2 \mathrm{~nm}$ respectively (Figure 2a). The zeta potential of the NPs was evaluated to confirm the polymer substitution and stability. The zeta potential of the PNP was negatively charged at $-23.67 \pm 3.21$ because of the carboxylic groups. The zeta potential changed to $21.37 \pm 1.28$ after coating with cationic polymer with an active amine functional group.

\section{Morphology}

The morphology of both NPs was evaluated using TEM and FE-SEM.

\section{DSC thermograms}

DSC was performed to assess the physical state of the drug after encapsulation in the NP as the physical state could influence the in vitro and in vivo properties of the drug. Figure 3 shows the DSC thermograms of pure DTX, ALN, PNP, and CPNP.

The melting endothermic peak of pure DTX appeared at $173{ }^{\circ} \mathrm{C}$, while that of ALN appeared at $128{ }^{\circ} \mathrm{C}$, indicating its sharp crystalline nature. The melting peak of both drugs was not detected in the NP formulation, indicating that the drugs were successfully incorporated into the NPs and present in the molecular dispersed and amorphous forms. It is worth noting that the crystalline form of the drug is not active and is not effective or stable in the systemic circulation. The amorphous form of the drug, however, circulates freely [13].

\section{In vitro drug release}

The release of DTX and ALN from CPNP was performed in PBS $(\mathrm{pH} 7.4)$ to simulate the physiological conditions of the human body. An initial release of approximately $\sim 30 \%$ from the NPs was observed in the first $8 \mathrm{~h}$, suggesting that a significant portion of the drug was present on the surface of the NP, which was released upon contact with the medium (Figure 4). Following this initial release, a more sustained release of the drug was observed for up to $48 \mathrm{~h}$, demonstrating the capacity of the carrier to withhold the drug while in systemic circulation. 

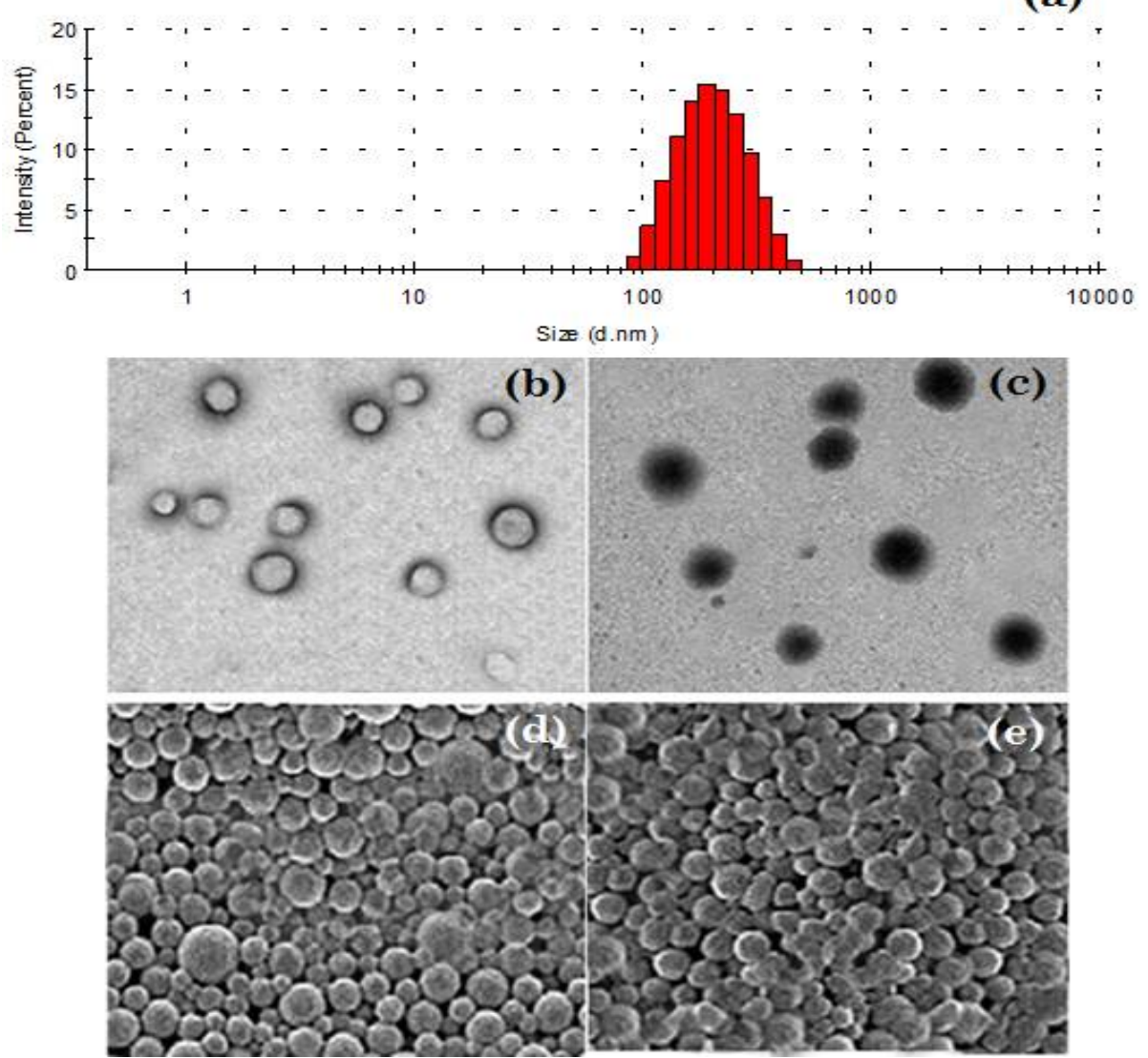

Figure 2: (a) Particle size distribution of chitosan (CS)-coated polylactide-co-glycolide nanoparticles CPNP; (b,c) transmission electron microscope (TEM)TEM images of polylactide-co-glycolide (PLGA NPS) and CS-conjugated PLGA NPs; (d,e) scanning electron microscope (SEM) images of PLGA NPs and CS-conjugated PLGA NPs. Panels $b$ and $c$ present TEM images of PNP and CPNP in the dried state, where PNPs are spherical in shape and uniformly distributed on the TEM grid. The particle shape was further confirmed by field emission scanning electron microscopy (FE-SEM) (c, d). PNP and CPNP were spherical in shape but with different surface textures

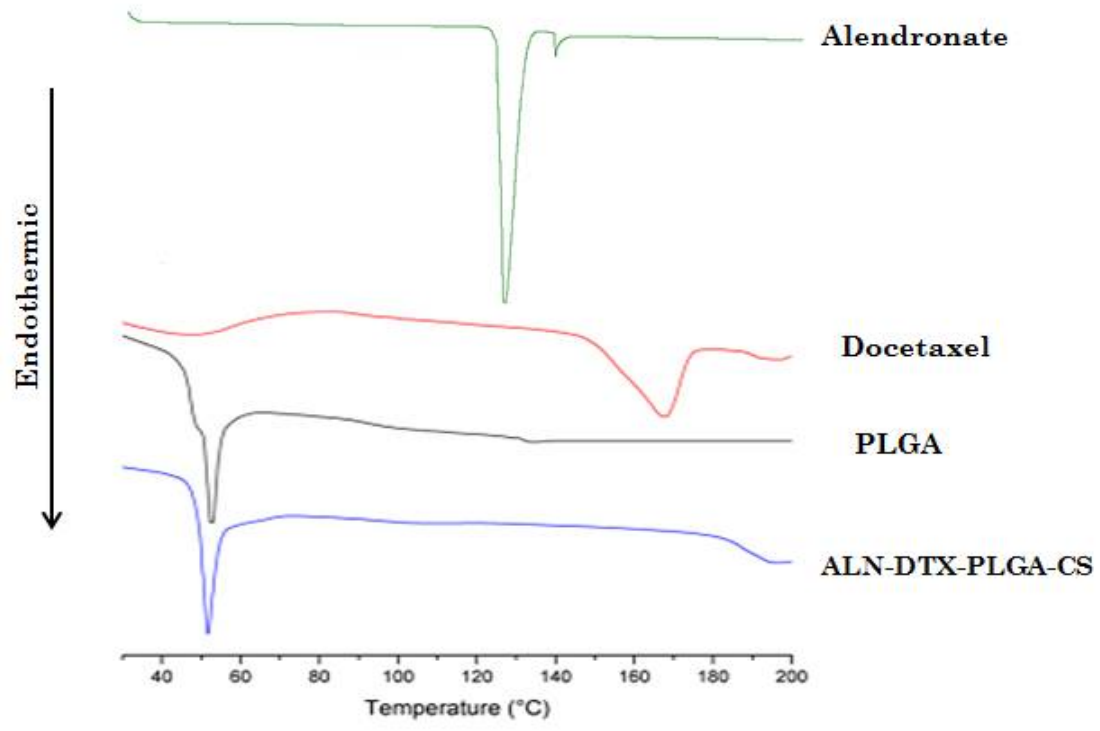

Figure 3: Differential scanning colorimetry (DSC) thermograms of free ALN, DTX, PLGA, and CPNP 


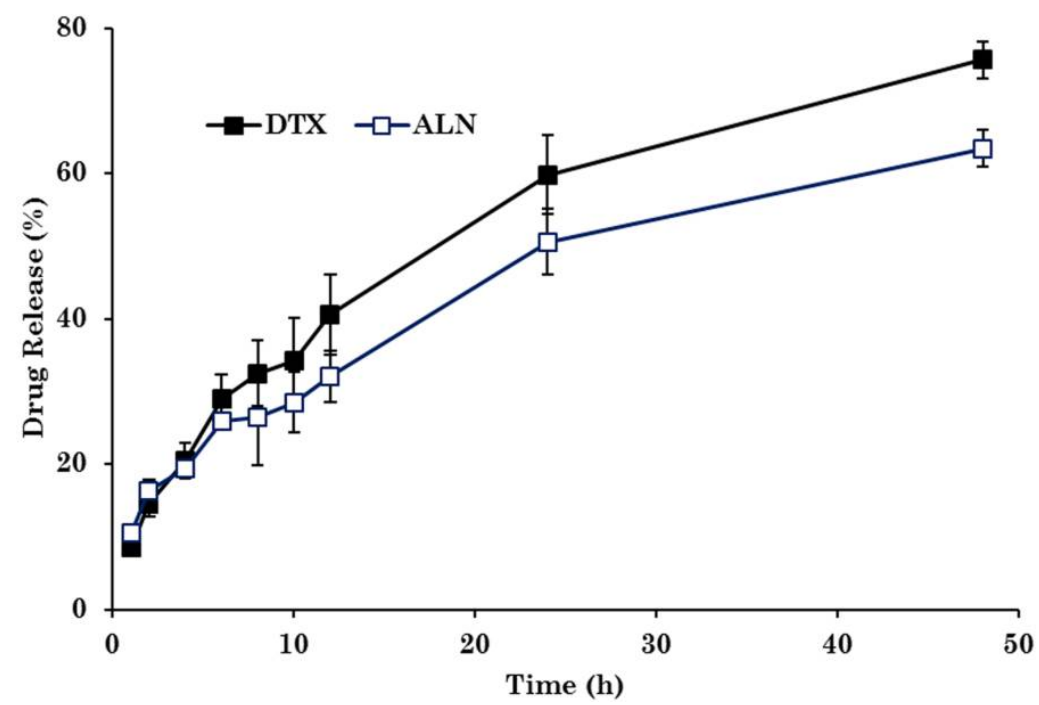

Figure 4: Drug release of ALN and DTX from chitosan (CS)-coated polylactide-co-glycolide nanoparticles $(\mathrm{CPNP})$ in release buffer CPNPs in PBS

(a)
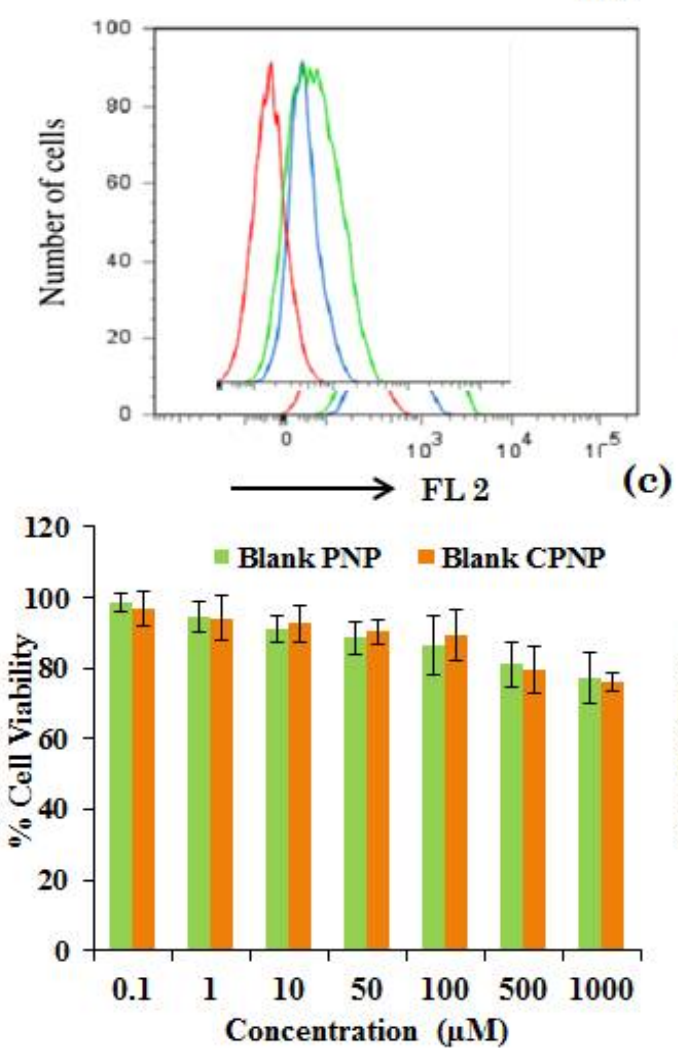

(b)

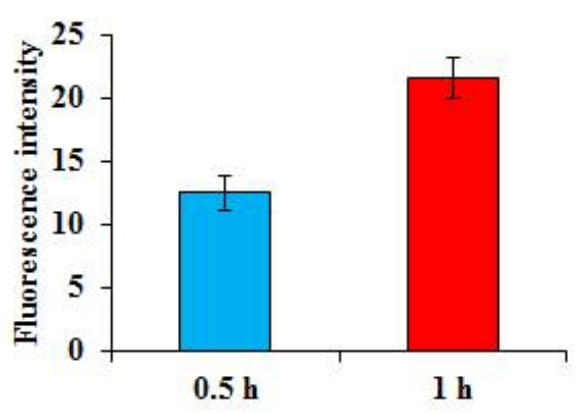

(d)

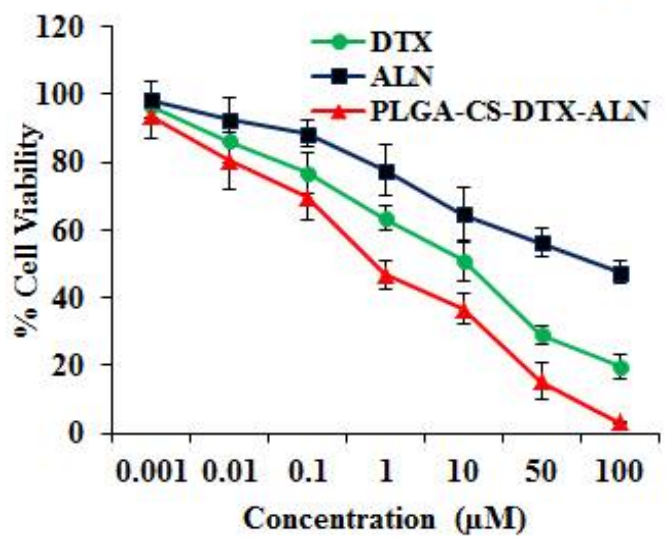

Figure 5: Flow cytometry analysis of cellular uptake (a, b) of PNPs and CPNPs in osteosarcoma cancer cells; (c) cytotoxicity assay of blank nanoparticles; (d) cytotoxicity assay of free drugs and drug-loaded nanoparticles

\section{Cellular uptake}

Flow cytometry analysis was performed to confirm the cellular uptake of CPNPs in MG-63 cancer cells. To accomplish this, the drug was replaced with the fluorescent probe Rhodamine B. A remarkable increase in fluorescence intensity was observed after 30-min incubation
(Figure 5a, b). Cellular uptake increased further when the incubation time was extended to $1 \mathrm{~h}$.

\section{In vitro cytotoxicity}

The in vitro cytotoxicity of blank PLGA NPs and blank CS-coated PLGA NPs was investigated in MG-63 cancer cells. Cancer cells were exposed 
to the polymers $(0.1-1,000 \mu \mathrm{g} / \mathrm{mL})$. Blank NP treatment resulted in high cell viability, suggesting that the polymers have excellent biocompatibility (Figure 5c, d). The lack of carrier cytotoxicity is advantageous for cancer cell targeting. MG-63 cells were also treated with free DTX, ALN, and DTX/ALN-loaded combined NPs. Both DTX and ALN treatment resulted in typical concentration-dependent cytotoxicity after $24 \mathrm{~h}$ incubation. Moreover, DTX was more effective than ALN in killing cancer cells.

\section{DISCUSSION}

First, DTX- and ALN-loaded PLGA NPs were prepared, and the carboxylic groups of PLGA NPs were conjugated to CS by an EDC/NHS reaction. PLGA NPs have a reduced affinity for cancer cells due to the negatively charged carboxyl groups on the PLGA surface. CS was therefore introduced to modify the surface charge, and improve chemotherapeutic efficacy. The positively charged NP system thus increased cellular uptake efficiency. The increase in the size of NP was attributed to the presence of the high molecular weight CS on the NP surface. It also indicated a definite deposition of mass on the NPs. The zeta potential changed to a positive charge after coating with a cationic polymer with an active amine functional group. The presence of this positive charge was expected to enhance the interaction between the cancer cells and NPs and facilitate cellular uptake [12].

The spherical nature of the NP was attributed to the self-assembly of the polymer in the presence of surfactant. CPNPs are also spherical particles with monodispersion, although the overall particle size is larger. The greyish shell on the NP surface might have been the CS coating, which increased the particle size. PNPs initially consisted of a smooth surface, while the CS coating slightly roughened the surface. Nevertheless, a spherical-shaped particle in the nanodimension is ideal for cancer tissue targeting.

Importantly, no significant difference in the release pattern of DTX and ALN was observed, indicating that the substitution of CS on the NP surface did not influence drug release from the delivery system [13]. The release data were fit to mathematical models, and the release kinetics were confirmed by fitting to the Higuchi model $(r$ $=0.999$ ), with the release profile indicating a diffusion-based release. A controlled release system in which the drug is released to the tumour tissue at a steady rate is of significant importance.
High cellular uptake efficiency is also necessary for the successful delivery of the drug to the tumour. Our results suggest that positively charged nanocarriers could be efficiently internalized in cancer cells. DTX/ALN-loaded CPNPs exhibited a superior cancer killing effect compared with either drug alone. Interestingly, introduction of DTX/ALN-loaded CPNPs resulted in only $45 \%$ cell viability, indicating its cytotoxicity. The $\mathrm{IC}_{50}$ value, or the concentration required to kill $50 \%$ of cancer cells, was investigated: the $\mathrm{IC}_{50}$ value of DTX/ALN-loaded CPNPs was nearly 20 times lower than that of the free drugs [14].

\section{CONCLUSION}

A unique DTX- and ALN-conjugated CS-coated PLGA nanoparticle formulation was prepared and evaluated for its potential in targeting osteosarcoma cells. The two-drug-loaded NP showed a superior cancer killing effect in MG-63 cancer cells and was safe and biocompatible. Combined loading of DTX and ALN increased the therapeutic efficacy in osteosarcoma treatment. Thus, combinatorial drug regimens using nanocarriers hold promise for the effective treatment of osteosarcoma.

\section{DECLARATIONS}

\section{Acknowledgement}

The study was funded by Technical Grant of KaiFeng Central Hospital, China.

\section{Conflict of Interest}

No conflict of interest associated with this work.

\section{Contribution of Authors}

The authors declare that this work was done by the authors named in this article and all liabilities pertaining to claims relating to the content of this article will be borne by them.

\section{REFERENCES}

1. Broadhead ML, Clark JC, Myers DE, Dass CR, Choong PF. The molecular pathogenesis of osteosarcoma: a review. Sarcoma. 2011; article ID 959248: 959-248.

2. He JP, Hao Y, Wang $X L$, Yang $X J$, Shao JF, Guo FJ, Feng JX. Review of the molecular pathogenesis of osteosarcoma. Asian Pac. J Cancer Prev. 2014; 15: 5967-5976. 
3. Cotterill SJ, Wright CM, Pearce MS, Craft AW. Stature of young people with malignant bone tumors. Pediatr Blood Cancer. 2004; 42: 59-63.

4. Jia J, Zhu F, Ma X, Cao Z, Li Y, Chen YZ. Mechanisms of drug combinations: interaction and network perspectives. Nat Rev Drug Discov. 2009; 8: 111-128.

5. Ramasamy T, Kim J, Choi HG, Yong CS, Kim JO. Novel dual drug-loaded block ionomer complex micelles for enhancing the efficacy of chemotherapy treatments. J Biomed Nanotechnol. 2014; 10: 1304-1312.

6. Pradhan R, Poudel BK, Ramasamy T, Choi HG, Yong CS, Kim JO. Docetaxel-loaded polylactic acid-coglycolic acid nanoparticles: formulation, physicochemical characterization and cytotoxicity studies. J Nanosci Nanotechnol. 2013; 13: 5948-5956.

7. Ernsting MJ, Murakami M, Undzys E, Aman A, Press B, $L i S D$. A docetaxel carboxy-methylcellulose nanoparticle outperforms the approved taxane nano formulation, Abraxane, in mouse tumor models with significant control of metastases. J Control Rel. 2012; 162: 575581.

8. Bereson JR, Lipton A. Bisphosphonates in the treatment of malignant bone disease. Annu Rev Med. 1999; 50 : 237-248.
9. Lipton A. Emerging role of bisphosphonates in the clinicantitumor activity and prevention of metastasis to bone. Cancer Treat Rev. 2008; 34, suppl. 1, S25-30.

10. Danhier F, Ansorena E, Silva JM, Coco R, Breton AL, Preat V. PLGA-based nanoparticles: An overview of biomedical applications. J Control Rel. 2012; 161: 505522.

11. Wang $H$, ZhaoY, Wu Y, Hu Y L, Nan K, Nie G, Chen $H$. Enhanced antitumor efficacy by co-delivery of doxorubicin and paclitaxel with amphiphilic methoxy PEG-PLGA copolymer nanoparticles. Biomaterials. 2011; 32: 8281-8290.

12. Ruttala HB, Ko YT. Liposomal co-delivery of curcumin and albumin/paclitaxel nanoparticle for enhanced synergistic antitumor efficacy. Colloids Surf., B. 2015; 128: 419-426.

13. Karl S, Pritschow $Y$, Volcic M, Hacker S, Baumann B, Wiesmuller L, Debatin KM, Fulda S.. Identification of a novel pro-apopotic function of NF-kappaB in the DNA damage response. J Cell Mo Med. 2009; 13: 42394256.

14. Saad M, Garbuzenko OB, Minko T. Co-delivery of siRNA and an anticancer drug for treatment of multidrugresistant cancer. Nanomedicine. 2008; 3: 761-776. 